\title{
The effect of genistein on insulin resistance, inflammatory factors, lipid profile, and histopathologic indices in rats with polycystic ovary syndrome
}

\author{
Sasan Amanat ${ }^{1}$, Fatemeh Ashkar ${ }^{2,3}$, Mohammad Hassan Eftekhari ${ }^{3}$, Nader Tanideh ${ }^{4}$, Saeid Doaei $^{5}$, Maryam Gholamalizadeh ${ }^{6}$, \\ Farhad Koohpeyma ${ }^{7}$, Maral Mokhtari ${ }^{8}$ \\ 'Department of Nutrition, School of Health, Larestan University of Medical Sciences, Larestan; ${ }^{2}$ Food and Nutrition Research Center and ${ }^{3}$ Department \\ of Clinical Nutrition, School of Nutrition and Food Sciences, Shiraz University of Medical Sciences, Shiraz; ${ }^{4}$ Stem Cells Technology Research Center, Shiraz \\ University of Medical Sciences, Shiraz; ${ }^{5}$ Reproductive Health Research Center, Department of Obstetrics and Gynecology, Al-Zahra Hospital, School of \\ Medicine, Guilan University of Medical Sciences, Rasht; ${ }^{6}$ Student Research Committee, Cancer Research Center, Shahid Beheshti University of Medical \\ Sciences, Tehran; ${ }^{7}$ Department of Endocrinology, Endocrinology and Metabolism Research Center, Shiraz University of Medical Sciences, Shiraz; \\ ${ }^{8}$ Department of Pathology, School of Medicine, Shiraz University of Medical Sciences, Shiraz, Iran
}

Objective: Polycystic ovary syndrome (PCOS) is characterized by hyperandrogenism, irregular menstruation, ovulatory dysfunction, and insulin resistance. Recent studies have reported the possible role of phytoestrogens in PCOS. This animal study aimed to evaluate the effects of genistein on insulin resistance, inflammatory factors, lipid profile, and histopathologic indices on PCOS.

Methods: PCOS was induced by $1 \mathrm{mg} / \mathrm{kg}$ of letrozole in adult Sprague-Dawley rats. The rats then received normal saline (PCOS group), 150 $\mathrm{mg} / \mathrm{kg}$ of metformin, or $20 \mathrm{mg} / \mathrm{kg}$ of genistein dissolved in $1 \%$ methylcellulose solution for 42 days. Body weight, the glycemic and lipid profile, and inflammatory, antioxidative, and histopathological parameters were assessed at the end of the intervention.

Results: Treatment with genistein significantly alleviated the increased level of fasting blood insulin $(p=0.16)$ and the homeostatic model assessment of insulin resistance $(p=0.012)$. In addition, the genistein group had significantly lower levels of serum malondialdehyde $(p=0.039)$ and tumor necrosis factor-alpha $(p=0.003)$, and higher superoxide dismutase enzyme activity $(p<0.001)$. Furthermore, the histopathological analysis indicated that genistein administration led to an increase in luteinization and the development of fewer cysts $(p<0.05)$.

Conclusion: Biochemical and histopathological analyses indicated that genistein administration to rats with PCOS induced significant remission in oxidative, inflammatory, and glycemic and histopathologic parameters.

Keywords: Genistein; Inflammation; Insulin resistance; Metformin; Phytoestrogen; Polycystic ovary syndrome

Received: November 18, 2020 • Revised: April 1, 2021· Accepted: April 1, 2021 Corresponding author: Mohammad Hassan Eftekhari

Department of Clinical Nutrition, School of Nutrition and Food Sciences, Shiraz University of Medical Sciences, Shiraz, Iran

Tel: +98-71-3230-5410 Fax:+98-71-3233-2093 E-mail:h_eftekhari@yahoo.com

*This study was funded by Shiraz University of Medical Sciences, Shiraz, Iran (44587).

This is an Open Access article distributed under the terms of the Creative Commons Attribution Non-Commercial License (http://creativecommons.org/licenses/by-nc/4.0/) which permits unrestricted non-commercial use, distribution, and reproduction in any medium, provided the original work is properly cited.

\section{Introduction}

Polycystic ovary syndrome (PCOS) is one of the most prevalent gynecological diseases in the world. It has been estimated that 5.6\%$6.1 \%$ of fertile women around the world are affected by PCOS [1]. Women with PCOS suffer from hyperandrogenism, irregular menstruation, hirsutism, and insulin resistance (IR). IR in these patients is partly due to the mild systemic inflammation that frequently occurs in this syndrome [2]. An increase in proinflammatory cytokines, such as tumor necrosis factor-alpha (TNF-a), can lead to impaired insulin 
signaling by induction of serine phosphorylation of insulin receptor substrate 1 (IRS1) [3]. One of the main goals of the management of PCOS is reducing IR, which can worsen hyperandrogenism and induce further metabolic abnormalities [4-6].

Several medication strategies have been proposed to reduce IR, for which metformin is one of the most popular recommendations. Metformin enhances glucose homeostasis by inhibiting glucose secretion from hepatocytes and increasing peripheral glucose uptake [7]. Despite the effectiveness of metformin in lowering IR, its side effects such as gastrointestinal distress and interactions with other drugs have raised concerns regarding long-term usage [8]. Some environmental factors (e.g., genetics and nutrition) are also considered as risk factors for PCOS [9-11].

Recent studies have focused on using natural ingredients to reduce the symptoms of PCOS. Active substances, such as isoflavones, which are naturally present in some plants and especially soybean, have been considered as potential alternatives. Genistein, daidzein, and glycitein are the most prominent isoflavones that have been recently studied. Most biological activities of isoflavones are related to their antioxidant and anti-inflammatory properties [12-15]. At the same time, they are known as phytoestrogens, meaning that these compounds have structural similarities with estrogen. Moreover, some beneficial effects may be associated with improvements in insulin sensitivity and amelioration of the lipid profile [16-18].

Studies of animal models have identified favorable results of pure genistein, which could improve the lipid profile and IR [19,20]. Moreover, these changes were accompanied by the promotion of oxidative status and secretion of proinflammatory cytokines [19,21,22]. Romuald et al. [23] found that phytoestrogens supplementation significantly reduced total cholesterol levels and low-density lipoprotein (LDL) in rats. Zhang and Chi [24] investigated the associations of genistein activity with follicle-stimulating hormone receptor and luteinizing hormone receptor expression in rats with PCOS. They found that genistein could improve ovarian function, with increased luteinizing hormone receptor protein expression and decreased follicle-stimulating hormone receptor protein expression.

Genistein supplementation may attenuate the metabolic symptoms of PCOS through its anti-inflammatory and anti-diabetic activities. Therefore, this study aimed to assess the effect of genistein supplementation in comparison to metformin therapy on glycemic indices; the inflammatory, oxidative, and lipid profile; and histopathologic factors in PCOS-induced rats.

\section{Methods}

The study was conducted in accordance with the recommendations of the European Council Directive (86/609/EEC) on November
24,1986 , regarding the protection of animals used for experimental purposes (http://data.europa.eu/eli/dir/ 1986/609/oj). All procedures were approved by the Ethics Committee of Shiraz University of Medical Sciences, Shiraz, Iran (IR.SUMS.REC.1395.S932). Consent for publication was not applicable. Letrozole (Aburaihan Pharmaceutical, Tehran, Iran) was used to induce PCOS (1 mg/kg dissolved in normal saline).

\section{Experimental design}

In this study, 40 adult (10-12 weeks) female Sprague-Dawley rats were obtained from Shiraz University of Medical Sciences and were housed in a controlled environment (temperature, $22^{\circ} \pm 2^{\circ} \mathrm{C}$; relative humidity, 55\% $\pm 3 \%$; 12-hour light-dark cycle). All the rats were fed with a normal diet ad libitum. The selected rats had two or three regular estrous cycles during the 2 weeks of vaginal smear observation, and they were in the estrous phase of their reproductive cycle. PCOS was induced using $1 \mathrm{mg} / \mathrm{kg}$ of letrozole (Aburaihan Pharmaceutical) dissolved in normal saline. The controls and PCOS-induced rats were evaluated using vaginal smears to determine the irregularity of the estrous cycle and the appearance of persistent vaginal cornification as a symptom of follicular cysts in the ovary.

The rats were initially kept in steel cages for 1 week to acclimate to the new environment. Then, they were randomly divided into four groups (10 rats in each group) as follows: the control group comprised healthy rats, the PCOS group included PCOS-induced rats that received normal saline by gavage, the metformin group comprised PCOS-induced rats treated with $150 \mathrm{mg} / \mathrm{kg}$ of metformin (Shafapharma, Tehran, Iran) dissolved in normal saline by gavage [25], and the genistein group included PCOS-induced rats supplemented with 20 $\mathrm{mg} / \mathrm{kg}$ of genistein (DSM Co., Heerlen, the Netherlands) dissolved in $1 \%$ methylcellulose solution. The genistein dosage was determined based on the effective dose of genistein supplementation reported in previous studies [26,27].

\section{Study design}

This study was carried out for 63 days. The first 21 days were dedicated to induction of PCOS by letrozole and the remaining 42 days to the specific interventions. At the end of the study, the rats were kept fasting overnight and were prepared for sacrifice in the next morning. Ketamine $(100 \mathrm{mg} / \mathrm{kg})$ and xylazine $(10 \mathrm{mg} / \mathrm{kg})$ were used to anesthetize the rats before cardiocentesis, blood sampling, and ovary sampling. After centrifuging the blood samples at 4,000 rpm for 10 minutes, the serum was separated and stored at $-80^{\circ} \mathrm{C}$. The ovaries were cleaned of fat, weighed, and fixed in $10 \%$ formalin. All rats were weighed on days 1, 21 (after PCOS induction), and 63 (at the end of the study). 


\section{Assessment of serum biochemical parameters}

Fasting blood sugar (FBS), high-density lipoprotein cholesterol, LDL cholesterol, total cholesterol, and triglyceride levels were measured by commercial assay kits (Pars Azmoon, Tehran, Iran) using an Auto-analyzer (BT 1500; Biotecnica, Roma, Italy). In order to estimate superoxide dismutase (SOD) activity and total antioxidant capacity (TAC), enzymatic and colorimetric methods were used based on commercial kit instructions (ZellBio, Lonsee, Germany). In addition, serum malondialdehyde (MDA) was measured using the modified thiobarbituric acid method, as reported by Satoh et al. [28]. Finally, circulating TNF-a levels were determined using the enzyme-linked immunosorbent assay (ELISA) method based on factory instructions of the commercial kit (Diaclone, Besançon, France).

\section{Hormonal assay}

Regarding to estimate IR, the fasting insulin level was measured using ELISA kits (Mercodia, Sweden). The homeostatic model assessment of insulin resistance (HOMA-IR) was then calculated using the following equation: (fasting insulin $[\mathrm{mU} / \mathrm{L}] \times$ fasting blood glucose $[\mathrm{mg} / \mathrm{dL}]) / 405$.

\section{Histopathological examinations}

The harvested ovaries were fixed in 10\% formalin and firmly embedded in paraffin blocks before being sectioned at $5-\mu \mathrm{m}$ thickness. Hematoxylin and eosin-stained slides were prepared using the method proposed by Manneras et al. [29]. The slides were then examined for histopathological alterations via a light microscope
(Olympus CX31; Olympus, Tokyo, Japan). The histopathological slides were evaluated using different criteria including hyperplasia of theca cells, decreased number of corpora lutea, incomplete luteinization, capsular thickening, and numerous subcapsular follicular cysts, and they were scored as showing no changes, slight changes, or remarkable changes for each criterion.

\section{Statistical analysis}

The data were reported as mean \pm standard deviation. The study groups were compared using one-way analysis of variance followed by the Tukey multiple comparison test. All analyses were done using IBM SPSS ver. 19 (IBM Corp., Armonk, NY, USA). The significance level was set at 0.05 .

\section{Results}

\section{Body weight, ovarian weight, and biochemical analysis}

As presented in Figure 1, PCOS induction markedly increased the weight gain in comparison to the healthy group. Accordingly, the weight of the PCOS-induced rats (PCOS, metformin, and genistein groups) was significantly lower than that of the control group on day 21. At the end of the study, the body weight decreased in the metformin and genistein groups in comparison to the PCOS group, but the differences were not statistically significant. Ovarian weights in the PCOS and metformin groups were significantly higher than in the control group. The metformin and genistein groups had significantly lower ovarian weights than the PCOS groups (Figure 1).
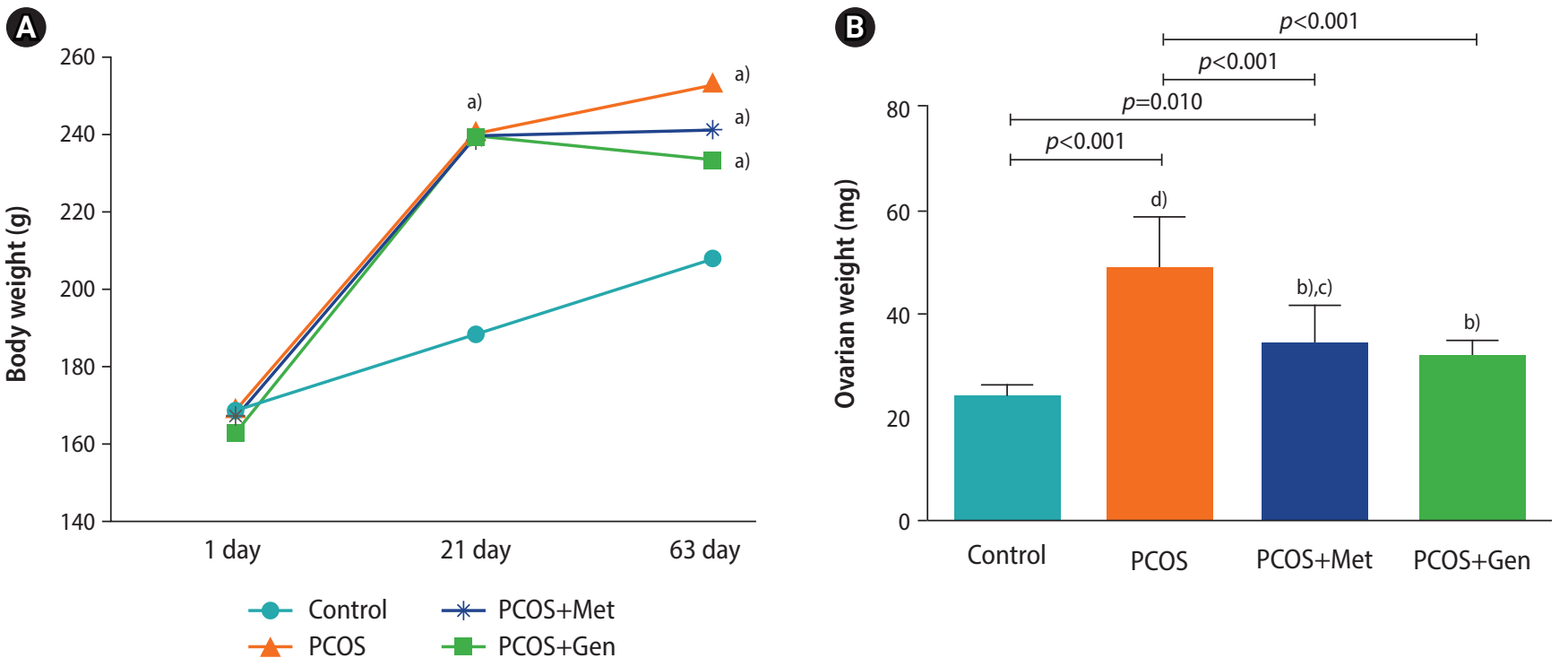

Figure 1. Changes in body weight (A) and ovarian weight (B) in the experimental groups. PCOS, polycystic ovary syndrome; Met, metformin $(150 \mathrm{mg} / \mathrm{kg})$; Gen, genistein $(20 \mathrm{mg} / \mathrm{kg})$. Statistically significant differences: ${ }^{\mathrm{a})} p<0.05$ and ${ }^{\mathrm{b})} p<0.001$, respectively, comparison with the PCOS group; ${ }^{c)} p<0.01$ and ${ }^{d)} p<0.001$, respectively, compared to the control group. 
As shown in Table 1, FBS increased significantly in the PCOS group, but not in the rats treated with metformin and genistein $(p=0.16)$. Similarly, fasting serum insulin levels increased only in the PCOS group 43 days after PCOS induction. Thus, PCOS induction was accompanied by an increase in IR in the PCOS group $(p=0.012)$. However, no significant increase was observed in HOMA-IR in the rats receiving metformin and genistein in comparison to the control group (Table 1). The results revealed no significant changes in circulating lipids in the study groups, except for LDL cholesterol, which increased significantly in the PCOS group compared to the control group and significantly decreased in the metformin- and genistein-treated rats compared to the PCOS group (Figure 2).

\section{Oxidative and inflammatory indices}

The PCOS group had a similar degree of SOD activity to the control group (Figure 3). However, the rats treated with genistein showed a significant increase in SOD activity compared to the healthy rats $(p<0.001)$. Moreover, TAC significantly decreased in the PCOS group compared to the control group, whereas TAC was maintained in both the metformin and genistein groups. In addition, lipid peroxidation as determined by MDA level was significantly elevated in the PCOS group $(p=0.01)$. A similar change was observed in the rats treated with met-

Table 1. The effects of metformin and genistein on glycemic indices in letrozole-induced PCOS

\begin{tabular}{lccl}
\hline Group & Insulin $(\mathrm{ng} / \mathrm{mL})$ & Glucose $(\mathrm{mg} / \mathrm{dL})$ & HOMA-IR \\
\hline Control & $0.87 \pm 0.09$ & $88.80 \pm 2.60$ & $3.45 \pm 0.43$ \\
PCOS & $1.75 \pm 0.34^{\text {a) }}$ & $121.66 \pm 5.51^{\text {a) }}$ & $9.34 \pm 1.80^{\text {a) }}$ \\
PCOS+Met & $1.18 \pm 0.20$ & $99.50 \pm 5.34^{\text {b) }}$ & $5.01 \pm 0.68^{\text {b) }}$ \\
PCOS+Gen & $1.01 \pm 0.14$ & $104.6 \pm 3.89^{\text {b) }}$ & $4.81 \pm 0.79^{\text {b) }}$ \\
\hline
\end{tabular}

Values are presented as mean \pm standard deviation.

HOMA-IR, homeostatic model assessment of insulin resistance; PCOS, polycystic ovary syndrome; Met, metformin (150 mg/kg); Gen, genistein ( $20 \mathrm{mg} / \mathrm{kg})$. ${ }^{\text {a) }} p<0.05$, PCOS group vs. control group; ${ }^{\text {b) }} p<0.05$, PCOS group vs. PCOS+ Met and PCOS+Gen groups.
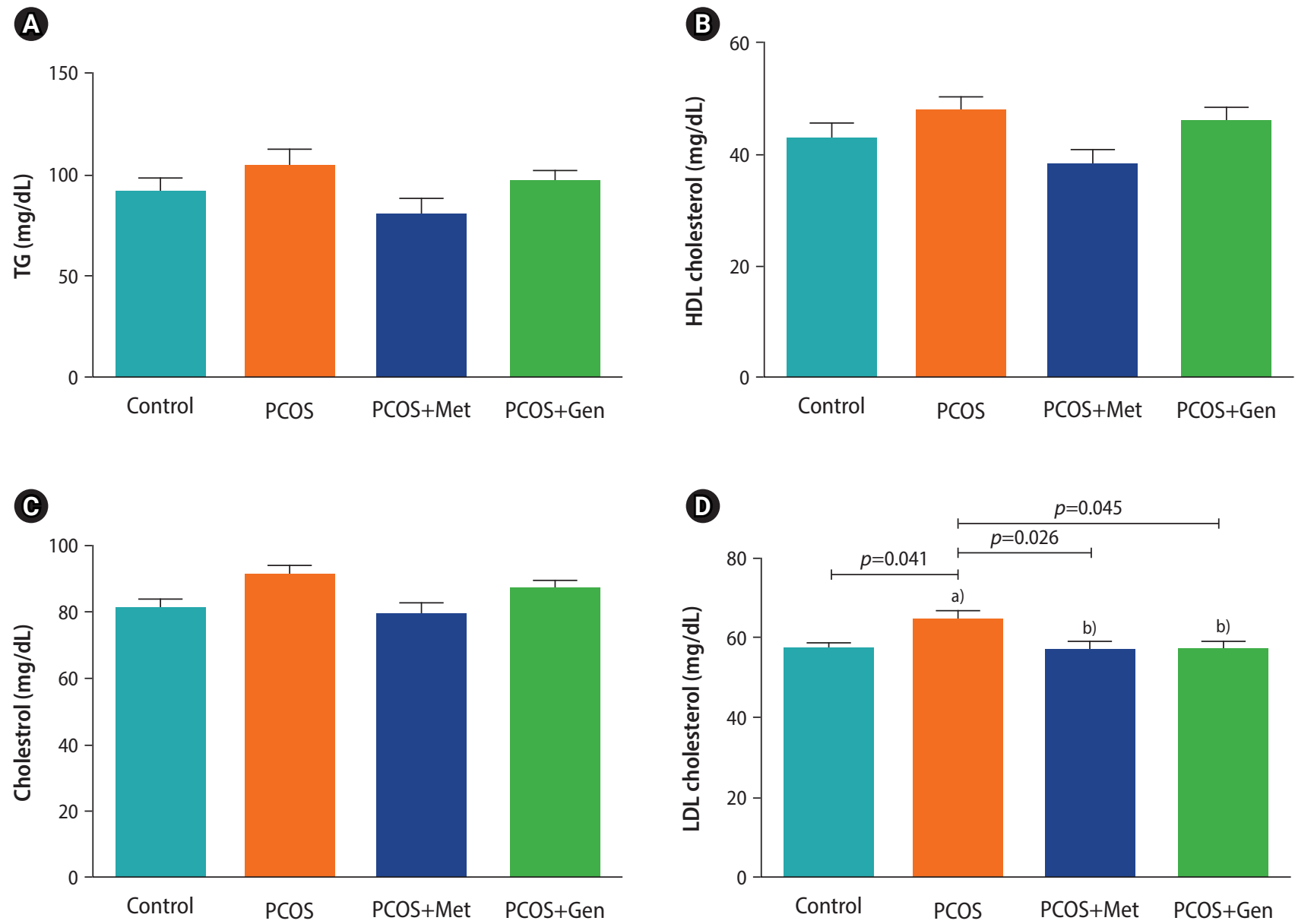

Figure 2. The effects of metformin and genistein therapy on lipid indices in the experimental groups. (A) Triglyceride (TG), (B) high-density lipoprotein (HDL) cholesterol, (C) cholesterol, (D) low-density lipoprotein (LDL) cholesterol. PCOS, polycystic ovary syndrome; Met, metformin $(150 \mathrm{mg} / \mathrm{kg})$; Gen, genistein $(20 \mathrm{mg} / \mathrm{kg})$. Statistically significant differences $(p<0.05)$ compared to ${ }^{\text {a) }}$ the control group and ${ }^{\mathrm{b})}$ the PCOS group. 
formin, while no change was observed in the genistein group. Furthermore, PCOS induction did not cause a higher degree of inflammation. Conversely, genistein supplementation reduced the levels of serum proinflammatory cytokines, such as TNF-a $(p=0.003)$, compared to the healthy rats.

\section{Histopathological changes}

Ovarian sections from the control group showed healthy follicles with oocytes at different developmental stages (Figure 4A and E). Letrozole-treated rats exhibited numerous sub-capsular cysts with very thin or no granulosa layers, and the volume of corpora lutea significantly decreased. In addition, there were few follicles in the ovaries of this group, which were mostly characterized by atresia (Figure $4 \mathrm{~B}$ and F). Treatment with metformin (Figure $4 \mathrm{C}$ and $\mathrm{G}$ ) and genistein
(Figure 4D and $\mathrm{H}$ ) led to a significant decrease in the number of cysts $(p<0.05)$ and the appearance of healthy follicles and corpora lutea. However, the number of atretic follicles was still significantly higher in these groups than in the control group.

\section{Discussion}

The results of the present study identified a greater weight gain in the PCOS-induced groups, which could be attributed to fat accumulation induced by IR, hepatic lipogenesis [30], and the higher level of androgens in PCOS [2]. Treatment with metformin and genistein did not prevent PCOS-induced weight gain, which might be related to the insulin-sensitizing activity of metformin and genistein [31,32].

In the present study, dysregulation of glucose hemostasis and IR in
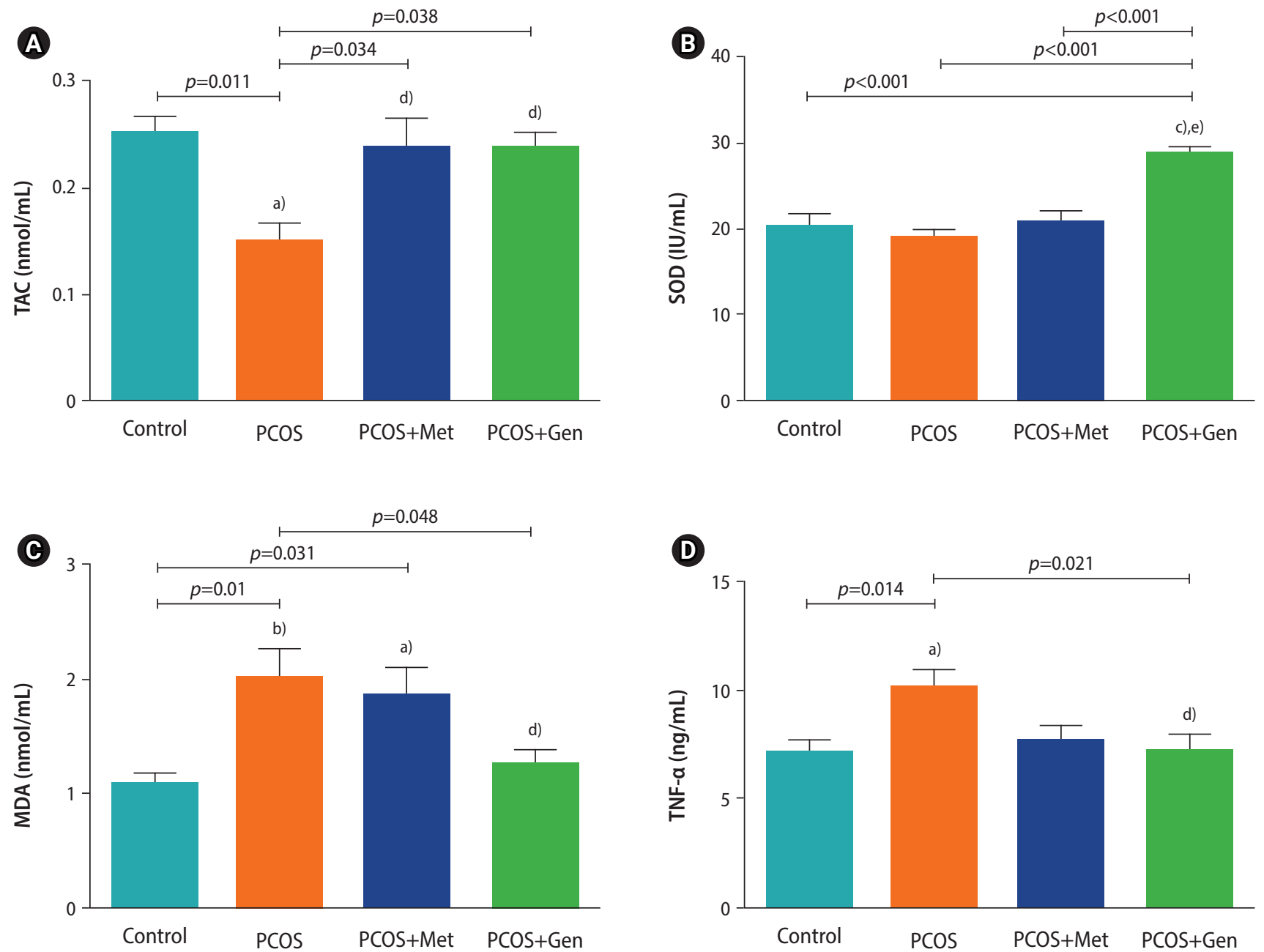

Figure 3. The effects of metformin and genistein treatment on oxidative and inflammatory indices in letrozole-induced PCOS. (A) Total antioxidant capacity (TAC), (B) superoxidase dismutase (SOD), (C) malondialdehyde (MDA), (D) tumor necrosis factor-alpha (TNF-a). PCOS, polycystic ovary syndrome; Met, metformin $(150 \mathrm{mg} / \mathrm{kg})$; Gen, genistein $(20 \mathrm{mg} / \mathrm{kg})$. Statistically significant differences: $\left.{ }^{\text {a) }} p<0.05\right),{ }^{\text {b) }} p<0.01$, and ${ }^{c)} p<0.001$ ), respectively, compared to the control group; ${ }^{\text {d) }} p<0.05$ and ${ }^{\text {e) }} p<0.001$ in comparison with the PCOS group. 
the PCOS group was significantly alleviated after genistein supplementation. It was reported that genistein reduced HOMA-IR mainly by decreasing fasting insulin levels in patients with non-alcoholic fatty liver disease [33]. This effect of genistein has been reported in several diseases $[18,33,34]$. Genistein may increase glucose uptake by augmentation of shifting glucose transporter type 4 (GLUT4) toward the cell membrane. It has been stated that phosphatidylinositol 3-kinase, protein kinase $C$, and 5 '-adenosine monophosphate-activated protein kinase (AMPK) pathways are involved in the effects of genistein on IR [35].

The histopathological slides in the genistein-supplemented rats showed fewer cysts, and the presence of corpora lutea in the ovaries indicated follicle maturation and ovulation. This aligns with the results of a previous study by Rajan et al. [36], in which treatment of PCOS-induced rats with $100 \mathrm{mg} / \mathrm{kg}$ of soy isoflavones caused the development of antral follicles and thickening of granulosa cell layer to the normal state. Interestingly, the glycemic and histopathological changes in the genistein group were fully comparable to those in the metformin-treated group.

Based on oxidative and antioxidant indices, PCOS induction led to higher oxidative status compared to the healthy group. This may itself be a cause of IR induction, as previously reported [37-39]. In addition, folliculogenesis and oocyte maturation were unfavorably affected by oxidative stress [40]. Genistein supplementation in the present study reversed TAC to the normal level and prevented lipid peroxide production by reactive oxygen species, while the metformin group had increased MDA levels. The effect of metformin on increasing TAC may be due to improvements in glucose metabolism, as explained previously. Similarly, genistein can augment antioxidant defenses by upregulating SOD activity. Genistein antioxidant activity mostly relies on two properties; first, the ROS scavenging activity of hydroxyl groups on the 4, 5, and 7 positions [41,42] and second, upregulation of gene expression for antioxidant enzymes (SOD, catalase, and glutathione peroxidase) [43]. The results of the current study confirmed the proposed mechanism, as the genistein group had significantly higher SOD activity than the PCOS and control groups. The current study demonstrated that PCOS induction significantly elevated levels of circulating proinflammatory cytokines, such as TNF-a. This low-grade, but chronic status of inflammation potentially increases the risk of metabolic disorders [44]. It has been reported that increased levels proinflammatory cytokines such as TNF- $a$ in PCOS are independent of body weight. However, the involved signaling pathways are similar, including increased serine phosphorylation, decreased insulin-stimulated IRS1 activation, and downregulation of GLUT4 expression $[3,45]$. The preservation of normal TNF-a levels in the genistein group may have been due to the anti-inflammatory function of genistein. A possible underlying mechanism could have been the effect of genistein in downregulation of nuclear factor kappa B (NF-kB) expression [46]. NF-кB is a transcription factor involved in the expression of several proinflammatory genes (e.g., TNF-a and interleukin-6) [47]. IR can substantially increase levels of circulating proinflammatory cytokines, which is similar to hyperinsulinemia/hyperandrogenism interaction in PCOS [48].

The present study revealed that no aspects of the lipid profile were
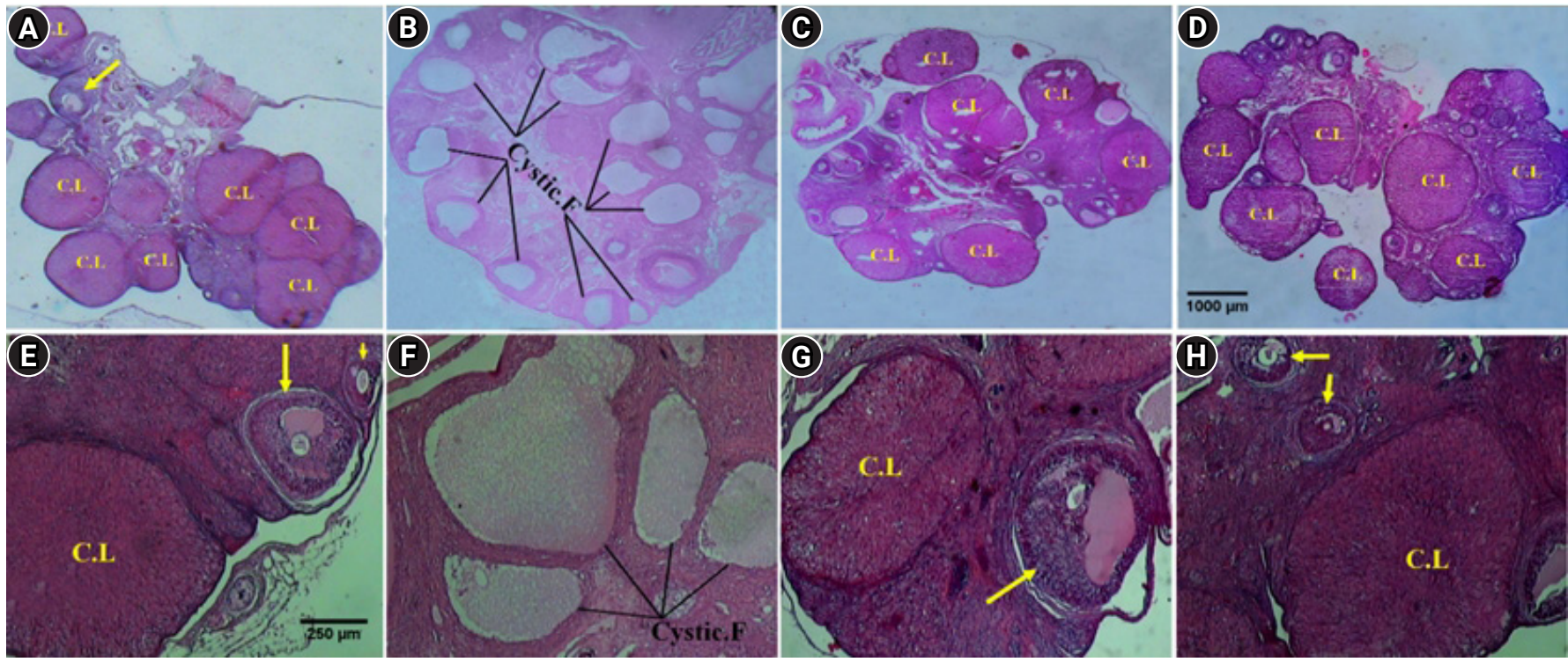

Figure 4. Photomicrograph of the rats' ovarian histopathology in different groups (arrows, antral follicles). (A, E) Control group, (B, F) polycystic ovary syndrome (PCOS) group, (C, G) PCOS+metformin group, (D, H) PCOS+genistein group. H\&E; magnification: $(A-D) \times 10$, (E-H) $\times 40$. CL, corpus luteum; F, cystic follicles. 
affected by letrozole, except for LDL cholesterol levels, which were increased significantly in the PCOS group. Metformin and genistein significantly suppressed this elevation to normal levels. The lipid-lowering action of metformin may be due to activation of hepatocytes via AMPK, which leads to the inhibition of $\beta$-Hydroxy $\beta$-methylglutaryl-CoA reductase [49]. However, the mechanism of genistein in LDL cholesterol reduction mostly relies on activation of peroxisome proliferator-activated receptor alpha, which subsequently decreases SREBP-2 expression and HMG-CoA reductase activity [50,51].

The promising results in the current study show that genistein may be a possible strategy to confront various complications of PCOS, including IR, dyslipidemia, and oxidative stress. However, further studies are required to investigate the effects of long-term usage of genistein in terms of favorable changes in biological features of PCOS such as hyperandrogenism and to identify its possible side effects. Changes in hormones, especially androgens, should also be examined in future studies.

This study demonstrated that genistein supplementation in rats with letrozole-induced PCOS mitigated IR. Moreover, the histopathological results indicated an increase in luteinization and the development of fewer cysts. These changes were comparable to those observed in the metformin-treated group. Moreover, both genistein and metformin therapy led to lower levels of LDL cholesterol, but only the rats receiving genistein exhibited improvements in antioxidant and anti-inflammatory status. Overall, the results revealed that genistein might be a safe and effective dietary component to ameliorate the complications of PCOS. In addition to acting as a phytoestrogen and regulating metabolic features, genistein exerts antioxidant and anti-inflammatory properties. If these possibilities are confirmed in future studies, genistein may be considered as a supplementary treatment in patients with PCOS.

\section{Conflict of interest}

No potential conflict of interest relevant to this article was reported.

\section{Acknowledgments}

The authors would like to thank the staff of the Clinical Nutrition Department of Shiraz University of Medical Sciences for their kind cooperation on the basis of this study.

\section{ORCID}

\section{Sasan Amanat}

Fatemeh Ashkar https://orcid.org/0000-0001-6848-6543

https://orcid.org/0000-0002-4921-4291
Mohammad Hassan Eftekhari https://orcid.org/0000-0001-5428-1491

NaderTanideh

https://orcid.org/0000-0001-9282-1778

https://orcid.org/0000-0002-2532-7478

https://orcid.org/0000-0001-8594-3574

https://orcid.org/0000-0001-6729-7732

https://orcid.org/0000-0002-4815-2631

\section{Author contributions}

Conceptualization: SA, FA, NT, MHE. Data curation: SA, FA, NT, FK. MM. Formal analysis: SA, SD, MG. Funding acquisition: FA, MHE. Methodology: SA, NT, SD. Project administration: SA, FA, NT, MHE. Visualization: FA, FK, MM, MG. Writing-original draft: SA, SD, MHE, NT. Writing-review \& editing: SD, MG, FK, MM, FA.

\section{References}

1. Ding T, Hardiman PJ, Petersen I, Wang FF, Qu F, Baio G. The prevalence of polycystic ovary syndrome in reproductive-aged women of different ethnicity: a systematic review and meta-analysis. Oncotarget 2017;8:96351-8.

2. Spritzer PM, Lecke SB, Satler F, Morsch DM. Adipose tissue dysfunction, adipokines, and low-grade chronic inflammation in polycystic ovary syndrome. Reproduction 2015;149:R219-27.

3. Gonzalez F. Inflammation in polycystic ovary syndrome: underpinning of insulin resistance and ovarian dysfunction. Steroids 2012;77:300-5.

4. Ciaraldi TP, Aroda V, Mudaliar S, Chang RJ, Henry RR. Polycystic ovary syndrome is associated with tissue-specific differences in insulin resistance. J Clin Endocrinol Metab 2009;94:157-63.

5. Corbould A. Chronic testosterone treatment induces selective insulin resistance in subcutaneous adipocytes of women. J Endocrinol 2007;192:585-94.

6. Ashkar F, Rezaei S, Salahshoornezhad S, Vahid F, Gholamalizadeh M, Dahka SM, et al. The role of medicinal herbs in treatment of insulin resistance in patients with polycystic ovary syndrome: a literature review. Biomol Concepts 2020;11:57-75.

7. DeFronzo RA, Barzilai N, Simonson DC. Mechanism of metformin action in obese and lean noninsulin-dependent diabetic subjects. J Clin Endocrinol Metab 1991;73:1294-301.

8. Huang Y, Sun J, Wang X, Tao X, Wang H, Tan W. Asymptomatic chronic gastritis decreases metformin tolerance in patients with type 2 diabetes. J Clin Pharm Ther 2015;40:461-5.

9. Shidfar F, Bahrololumi SS, Doaei S, Mohammadzadeh A, Gholamalizadeh M, Mohammadimanesh A. The effects of extra virgin olive oil on alanine aminotransferase, aspartate aminotransferase, and ultrasonographic indices of hepatic steatosis in nonalcoholic 
fatty liver disease patients undergoing low calorie diet. Can J Gastroenterol Hepatol 2018;2018:1053710.

10. Doaei S, Kalantari N, Keshavarz Mohammadi N, Izadi P, Gholamalizadeh $\mathrm{M}$, Eini-Zinab $\mathrm{H}$, et al. The role of FTO genotype in the association between FTO gene expression and anthropometric measures in obese and overweight adolescent boys. Am J Mens Health 2019;13:1557988318808119.

11. Doaei S, Hajiesmaeil M, Aminifard A, Mosavi-Jarrahi SA, Akbari ME, Gholamalizadeh M. Effects of gene polymorphisms of metabolic enzymes on the association between red and processed meat consumption and the development of colon cancer; a literature review. J Nutr Sci 2018;7:e26.

12. Kim HP, Son KH, Chang HW, Kang SS. Anti-inflammatory plant flavonoids and cellular action mechanisms. J Pharmacol Sci 2004;96: 229-45.

13. Serafini M, Miglio C, Peluso I, Petrosino T. Modulation of plasma non enzimatic antioxidant capacity (NEAC) by plant foods: the role of polyphenols. Curr Top Med Chem 2011;11:1821-46.

14. Heim KE, Tagliaferro AR, Bobilya DJ. Flavonoid antioxidants: chemistry, metabolism and structure-activity relationships. J Nutr Biochem 2002;13:572-84.

15. Lotfi MH, Fallahzadeh H, Rahmanian M, Hosseinzadeh M, Lashkardoost H, Doaei S, et al. Association of food groups intake and physical activity with gestational diabetes mellitus in Iranian women. J Matern Fetal Neonatal Med 2020;33:3559-64.

16. Zaheer K, Humayoun Akhtar M. An updated review of dietary isoflavones: nutrition, processing, bioavailability and impacts on human health. Crit Rev Food Sci Nutr 2017;57:1280-93.

17. Gilbert ER, Liu D. Anti-diabetic functions of soy isoflavone genistein: mechanisms underlying its effects on pancreatic $\beta$-cell function. Food Funct 2013;4:200-12.

18. Mohamed Salih S, Nallasamy P, Muniyandi P, Periyasami V, Carani Venkatraman A. Genistein improves liver function and attenuates non-alcoholic fatty liver disease in a rat model of insulin resistance. J Diabetes 2009;1:278-87.

19. Incir S, Bolayirli IM, Inan O, Aydin MS, Bilgin IA, Sayan I, et al. The effects of genistein supplementation on fructose induced insulin resistance, oxidative stress and inflammation. Life Sci 2016;158: 57-62.

20. Fu Z, Gilbert ER, Pfeiffer L, Zhang Y, Fu Y, Liu D. Genistein ameliorates hyperglycemia in a mouse model of nongenetic type 2 diabetes. Appl Physiol Nutr Metab 2012;37:480-8.

21. Yoo NY, Jeon S, Nam Y, Park YJ, Won SB, Kwon YH. Dietary supplementation of genistein alleviates liver inflammation and fibrosis mediated by a methionine-choline-deficient diet in $\mathrm{db} / \mathrm{db}$ Mice. J Agric Food Chem 2015;63:4305-11.

22. Susutlertpanya W, Werawatganon D, Siriviriyakul P, Klaikeaw N.
Genistein attenuates nonalcoholic steatohepatitis and increases hepatic PPARy in a rat model. Evid Based Complement Alternat Med 2015;2015:509057.

23. Romualdi D, Costantini B, Campagna G, Lanzone A, Guido M. Is there a role for soy isoflavones in the therapeutic approach to polycystic ovary syndrome? Results from a pilot study. Fertil Steril 2008;90:1826-33.

24. Zhang T, Chi XX. Estrogenic properties of genistein acting on FSHR and LHR in rats with PCOS. Pol J Vet Sci 2019;22:83-90.

25. Lv WS, Wen JP, Li L, Sun RX, Wang J, Xian YX, et al. The effect of metformin on food intake and its potential role in hypothalamic regulation in obese diabetic rats. Brain Res 2012;1444:11-9.

26. Chi XX, Zhang T, Chu XL, Zhen JL, Zhang DJ. The regulatory effect of Genistein on granulosa cell in ovary of rat with PCOS through Bcl-2 and Bax signaling pathways. J Vet Med Sci 2018;80:134855.

27. Liu H, Zhong H, Leng L, Jiang Z. Effects of soy isoflavone on hepatic steatosis in high fat-induced rats. J Clin Biochem Nutr 2017; 61:85-90

28. Satoh K, Takamatsu S, Sakuta S, Mizuno S, Metoki H, Takamatsu M. Augmented malondialdehyde production by platelets from patients with cerebrovascular disorders. Jpn Circ J 1981;45:1335-41.

29. Manneras L, Cajander S, Holmang A, Seleskovic Z, Lystig T, Lonn M, et al. A new rat model exhibiting both ovarian and metabolic characteristics of polycystic ovary syndrome. Endocrinology 2007;148: 3781-91.

30. Ben Djoudi Ouadda A, Levy E, Ziv E, Lalonde G, Sane AT, Delvin E, et al. Increased hepatic lipogenesis in insulin resistance and type 2 diabetes is associated with AMPK signalling pathway up-regulation in Psammomys obesus. Biosci Rep 2009;29:283-92.

31. Choi JS, Song J. Effect of genistein on insulin resistance, renal lipid metabolism, and antioxidative activities in ovariectomized rats. Nutrition 2009;25:676-85.

32. Simperova A, Al-Nakkash L, Faust JJ, Sweazea KL. Genistein supplementation prevents weight gain but promotes oxidative stress and inflammation in the vasculature of female obese ob/ob mice. Nutr Res 2016;36:789-97.

33. Choi JS, Koh IU, Song J. Genistein reduced insulin resistance index through modulating lipid metabolism in ovariectomized rats. Nutr Res 2012;32:844-55.

34. Palanisamy N, Viswanathan P, Anuradha CV. Effect of genistein, a soy isoflavone, on whole body insulin sensitivity and renal damage induced by a high-fructose diet. Ren Fail 2008;30:645-54.

35. Ha BG, Nagaoka M, Yonezawa T, Tanabe R, Woo JT, Kato H, et al. Regulatory mechanism for the stimulatory action of genistein on glucose uptake in vitro and in vivo. J Nutr Biochem 2012;23:501-9. 36. Rajan RK, Kumar SS, Balaji B. Soy isoflavones exert beneficial ef- 
fects on letrozole-induced rat polycystic ovary syndrome (PCOS) model through anti-androgenic mechanism. Pharm Biol 2017;55: 242-51.

37. Gonzalez F, Rote NS, Minium J, Kirwan JP. Reactive oxygen species-induced oxidative stress in the development of insulin resistance and hyperandrogenism in polycystic ovary syndrome. J Clin Endocrinol Metab 2006;91:336-40.

38. Sabuncu T, Vural H, Harma M, Harma M. Oxidative stress in polycystic ovary syndrome and its contribution to the risk of cardiovascular disease. Clin Biochem 2001;34:407-13.

39. Fenkci V, Fenkci S, Yilmazer M, Serteser M. Decreased total antioxidant status and increased oxidative stress in women with polycystic ovary syndrome may contribute to the risk of cardiovascular disease. Fertil Steril 2003;80:123-7.

40. Agarwal A, Gupta S, Sharma R. Oxidative stress and its implications in female infertility: a clinician's perspective. Reprod Biomed Online 2005;11:641-50.

41. Choi JN, Kim D, Choi HK, Yoo KM, Kim J, Lee CH. 2'-Hydroxylation of genistein enhanced antioxidant and antiproliferative activities in mcf-7 human breast cancer cells. J Microbiol Biotechnol 2009; 19:1348-54.

42. Sherif HS, Gebreyohannes B. Synthesis, characterization, and antioxidant activities of genistein, biochanin a, and their analogues. J Chem 2018;2018:4032105.

43. Borras C, Gambini J, Gomez-Cabrera MC, Sastre J, Pallardo FV, Mann GE, et al. Genistein, a soy isoflavone, up-regulates expression of antioxidant genes: involvement of estrogen receptors, ERK1/2, and NFkappaB. FASEB J 2006;20:2136-8.

44. Pawelczak M, Rosenthal J, Milla S, Liu YH, Shah B. Evaluation of the pro-inflammatory cytokine tumor necrosis factor-a in adolescents with polycystic ovary syndrome. J Pediatr Adolesc Gynecol 2014;27:356-9.

45. Corbould A, Kim YB, Youngren JF, Pender C, Kahn BB, Lee A, et al. Insulin resistance in the skeletal muscle of women with PCOS involves intrinsic and acquired defects in insulin signaling. Am J Physiol Endocrinol Metab 2005;288:E1047-54.

46. Ji G, Yang Q, Hao J, Guo L, Chen X, Hu J, et al. Anti-inflammatory effect of genistein on non-alcoholic steatohepatitis rats induced by high fat diet and its potential mechanisms. Int Immunopharmacol 2011;11:762-8.

47. Pearson G, English JM, White MA, Cobb MH. ERK5 and ERK2 cooperate to regulate NF-kappaB and cell transformation. J Biol Chem 2001;276:7927-31.

48. Shorakae S, Ranasinha S, Abell S, Lambert G, Lambert E, de Courten $B$, et al. Inter-related effects of insulin resistance, hyperandrogenism, sympathetic dysfunction and chronic inflammation in PCOS. Clin Endocrinol (Oxf) 2018;89:628-33.

49. Henin N, Vincent MF, Gruber HE, Van den Berghe G. Inhibition of fatty acid and cholesterol synthesis by stimulation of AMP-activated protein kinase. FASEB J 1995;9:541-6.

50. Kim S, Shin HJ, Kim SY, Kim JH, Lee YS, Kim DH, et al. Genistein enhances expression of genes involved in fatty acid catabolism through activation of PPARalpha. Mol Cell Endocrinol 2004; 220:51-8.

51. Konig B, Koch A, Spielmann J, Hilgenfeld C, Stangl Gl, Eder K. Activation of PPARalpha lowers synthesis and concentration of cholesterol by reduction of nuclear SREBP-2. Biochem Pharmacol 2007;73:574-85. 